\title{
The Index and Stock Price Synchronicity: Evidence from Taiwan
}

\author{
Chung-Lien Pan ${ }^{1}$, Yu-Chun Pan ${ }^{2 *}$ \\ ${ }^{1}$ Accounting School, Nanfang College of Sun Yat-sen University, Guangzhou, Guangdong, 510970, China \\ ${ }^{2}$ Graduate Institute of Finance, National Taiwan University of Science and Technology, Taipei, 10607, \\ Taiwan
}

\begin{abstract}
Research on stock synchronization has always been a topic of concern to scholars and investors. In the past, the focus was mainly on equity concentration, foreign shareholding, audit quality, and other issues, not including indexes. This article uses the monthly data of the Taiwan Stock Exchange Capital Weighted Stock Index (TAIEX) to solve the problem of the index and stock synchronization. And use the technical theory of the gray system to solve the small sample and uncertain problem. The discovery of the synchronization between these indexes and stock prices may provide investors with sufficient reference to make investment decisions.
\end{abstract}

\section{Introduction}

Investors seek to avoid irrational and blind investment behavior by adopting an optimal investment strategy. They have attempted to use various instruments to identify the pattern of changes in the stock market, predict future trends in share prices, and improve the timing of buying and selling. Investors have a difficult time doing this because of the complexity of the stock market. So Lee and Jo (1999) [1]showed that the timing of buying/selling stock is based on determining the best time to buy and sell stocks given the constant fluctuation of stock prices.

It is difficult to predict stock prices because the volumes of data are too huge to influence the ability to use information (Fayyad et al., 1996 and Widom, 1995)[2][3]. A multi-year trend analysis of the stock price thus still presents a problem due to the vast amount of data involved. It is, therefore, important to devise efficient methods for the analysis and predict stock prices. For this reason, we constructed a data mart (Demarest, 1994)[4], a relational database, to clean and reduce the size of the stock data so only the useful data is downloaded into the data mart (Liu \& Setiono, 1996)[5].

They showed that many studies address the prediction of the stock price that has generally employed the time series analysis techniques and multiple regression models. But they only consider quantitative factors like technical indexes.

The grey theory that was first proposed by avoids the inherent defects of conventional statistic methods and only requires a limited amount of data to estimate the behavior of unknown systems (Deng 1982 and 1989a,b)[6][7].
In this paper, we use his theory to solve the change associated with the stock and index. The current research on stock synchronization covers the following topics: equity concentration, foreign shareholding, audit quality, system development, trade secret law, trust, analyst coverage, but does not include indexes (Chan and Hameed (2006), Gul, Kim et al. (2010), Hasan, Song et al. (2014), $\mathrm{Kim}, \mathrm{Su}$ et al. (2020) and Qiu, $\mathrm{Yu}$ et al. (2019)[8][9][10][11][12].

\section{Data and methods}

\subsection{Data for association problem within grey system theory}

The constituent stocks of the FTSE Taiwan 50 Index of the Taiwan Stock Exchange account for nearly $70 \%$ of the market capitalization of the Taiwan market and are the highest blue-chip stocks. The benchmark period of the Taiwan 50 Index began on April 30, 2002. Quarterly audits of constituent stocks are conducted in March, June, September, and December each year. The core audit criteria are market value, free float, and liquidity. This article takes these 50 securities as the research sample which is the most representative for Taiwan's securities market.

For the completeness and correctness of the information, this article applies the above audit standards to collect data between January 2003 and December 2018 as test samples. Any stocks with incomplete data during the study period and stocks replaced by stocks on the reserve list are eliminated. The final sample structure is composed of 43 constituent stocks. The data uses the

*Corresponding author's e-mail: peter5612@gmail.com 
closing price of the first trading day of each month to measure the synchronization of stocks.

\section{2 research methods}

Many researchers have conducted much of the research to measure the change of index associate with the stock.
According to the methodology used by researchers, previous studies can be divided into three streams of research: one stream of research using statistical methods and the other streams of research using the fuzzy stock prediction system and grey theory. The comparison of the three research methods is shown in Table 1.

This research uses gray theory to solve the problem of stock price synchronization.

Table 1 Difference of Probability Statistics, Fuzzy Theory, Gray Theory

\begin{tabular}{|c|c|c|c|}
\hline $\begin{array}{l}\text { Comparison Of } \\
\text { Projects And } \\
\text { Differences }\end{array}$ & Probability Statistics & Fuzzy Theory & Gray Theory \\
\hline 1.Essential & Large Sample and & Uncertainty of & Small Sample and \\
\hline Connotation & Uncertain & Cognition & Uncertain \\
\hline 2.Mathematical Basis & Cantor Set & Fuzzy Set & Hazy Set \\
\hline $\begin{array}{l}\text { 3.Mathematical } \\
\text { Operation }\end{array}$ & Statistical Methods & $\begin{array}{l}\text { Take the Boundary } \\
\text { Value }\end{array}$ & Generating Method \\
\hline 4.How Much Data & Multiple Data State & Empirical Data Status & Less Data State \\
\hline 5.Data distribution & Typical Distribution & $\begin{array}{l}\text { The Distribution of } \\
\text { Functions }\end{array}$ & Arbitrary Distribution \\
\hline $\begin{array}{l}\text { 6.The Goal Of } \\
\text { Completion }\end{array}$ & $\begin{array}{l}\text { The Statistical Law of } \\
\text { History }\end{array}$ & Cognitive Expression & The Law of Reality \\
\hline
\end{tabular}

\section{RESEARCH MAPPING RESULTS}

\subsection{Calculate the average value of the relationship coefficient, from the index and constituent stocks}

According to the technical theory of the gray system, this paper uses six steps to calculate the average value of the correlation coefficient between the index and the constituent stocks.

First, the reference sequence $\mathrm{A} 0$ and the comparison sequence Ai must be determined. The combination of ideal target values for each influencing factor is set as the reference order, and the composition of each suggested performance value is set as the comparison order. In this article, the reference sequence is set as an index, and the comparison sequence is 43 constituent stocks.
Second, in order to simplify the calculation of the data, this paper standardized the values of the collected series. The denominator is defined as the closing price of the index and 216 constituent stocks on January 2, 2003. And sort by month, calculate the percentage of other 215 test samples. Place the index of the reference sequence in the first row, and rank individual stocks in other comparison sequences from the second row to the 44th row to complete the numerical standardization work.

The third step is the calculation of the gray relationship distance $\Delta$ oij.

Fourth, calculate the gray correlation coefficient $\gamma$ oij.

Fifth, we calculated the grey relational grade $\Gamma$ oi.

Sixth, we addressed the grey relational rank. After calculating the standard data from step 3 to step 6 , the analysis results in Table 2 are obtained.

Table 2 Results of Grey Relational Analysis

\begin{tabular}{cccccc}
\hline Stock & $\begin{array}{c}\text { the Mean Values of } \\
\text { Relational } \\
\text { Coefficient }\end{array}$ & Sequence & Stock & $\begin{array}{c}\text { the Mean Values } \\
\text { of Relational } \\
\text { Coefficient }\end{array}$ & Sequence \\
\hline 1303 & 0.9979 & 1 & 2885 & 0.9855 & 23 \\
1301 & 0.9971 & 2 & 2408 & 0.9854 & 24 \\
2412 & 0.9951 & 3 & 2303 & 0.9848 & 25 \\
1326 & 0.9943 & 4 & 2882 & 0.984 & 26 \\
1402 & 0.9932 & 5 & 2892 & 0.984 & 26 \\
2105 & 0.9925 & 6 & 2880 & 0.983 & 28 \\
2002 & 0.9923 & 7 & 2890 & 0.9829 & 29 \\
1102 & 0.9922 & 8 & 2884 & 0.9828 & 30 \\
4904 & 0.9916 & 9 & 2409 & 0.9824 & 31 \\
2823 & 0.9913 & 10 & 2883 & 0.9822 & 32 \\
6505 & 0.9902 & 11 & 2308 & 0.981 & 33
\end{tabular}




\begin{tabular}{lccccc}
1101 & 0.99 & 12 & 2891 & 0.9808 & 34 \\
2881 & 0.9898 & 13 & 2887 & 0.9792 & 35 \\
2801 & 0.9886 & 14 & 2912 & 0.9791 & 36 \\
2317 & 0.9882 & 15 & 3045 & 0.9772 & 37 \\
2382 & 0.9881 & 16 & 2357 & 0.9731 & 38 \\
2886 & 0.9881 & 16 & 1216 & 0.9687 & 39 \\
2395 & 0.9879 & 18 & 2354 & 0.9604 & 40 \\
2301 & 0.9876 & 19 & 2474 & 0.9563 & 41 \\
2454 & 0.9875 & 20 & 2327 & 0.9441 & 42 \\
2330 & 0.9873 & 21 & 3008 & 0.9026 & 43 \\
9904 & 0.9871 & 22 & & & \\
\hline
\end{tabular}

\subsection{Synchronization verification between the index and constituent stocks}

To solve the problem of synchronization verification between the index and the constituent stocks, multiple calculations and numerical standardization must be carried out for the index and the 43 constituent stocks to calculate the average value of the relationship coefficient between each sample and the index. Finding the sample with the largest value means the stronger the relationship with exponential synchronization.

What is the meaning behind the ranking of the average correlation coefficient? This article cross-compared it with the market capitalization ratio rankings of TAIEX constituent stocks, and you can understand the importance of the correlation coefficient average series.

The analysis results after cross-comparison are shown in Table 3.

Table 3 Constituents in TWSE TAIEX, Proportion of Market Capitalization and Relational Coefficient

\begin{tabular}{cccc}
\multicolumn{3}{c}{ Sequence } \\
\hline Stock & Ranking of Stock & Ranking of & Market \\
Weight Value & Relational & Capitalization as a \\
& & Coefficient & Proportion of \\
& & & TAIEX \\
\hline 2330 & 1 & 21 & $19.298 \%$ \\
2317 & 2 & 15 & $3.3413 \%$ \\
6505 & 3 & 11 & $3.2779 \%$ \\
2412 & 4 & 3 & $2.7297 \%$ \\
1301 & 5 & 2 & $2.2697 \%$ \\
1303 & 6 & 1 & $1.9412 \%$ \\
1326 & 7 & 4 & $1.8799 \%$ \\
2882 & 8 & 26 & $1.6822 \%$ \\
3008 & 9 & 43 & $1.6103 \%$ \\
2454 & 10 & 20 & $1.5523 \%$ \\
1216 & 11 & 39 & $1.4633 \%$ \\
2881 & 12 & 13 & $1.4611 \%$ \\
3045 & 13 & 37 & $1.3098 \%$ \\
2886 & 14 & 16 & $1.3086 \%$ \\
2891 & 34 & $1.2962 \%$ \\
2308 & 15 & 33 & $1.274 \%$ \\
2002 & 16 & 7 & $1.2225 \%$ \\
2912 & 17 & 36 & $0.9728 \%$ \\
2884 & 18 & 30 & $0.8768 \%$ \\
2892 & 19 & 26 & $0.876 \%$ \\
4904 & 20 & 28 & $0.7945 \%$ \\
2880 & 24 & 12 & $0.7495 \%$ \\
1101 & 25 & & $0.7325 \%$ \\
& 26 & &
\end{tabular}




$\begin{array}{lccc}2382 & 27 & 16 & 0.7265 \% \\ 2885 & 29 & 23 & 0.6787 \% \\ 2801 & 30 & 14 & 0.6387 \% \\ 2408 & 31 & 24 & 0.6133 \% \\ 2395 & 32 & 18 & 0.5747 \% \\ 1402 & 33 & 5 & 0.5584 \% \\ 2474 & 34 & 41 & 0.5338 \% \\ 2303 & 35 & 25 & 0.5267 \% \\ 2357 & 37 & 38 & 0.5158 \% \\ 1102 & 39 & 8 & 0.4977 \% \\ 2887 & 40 & 35 & 0.4648 \% \\ 2890 & 41 & 29 & 0.458 \% \\ 2883 & 42 & 32 & 0.4413 \% \\ 2105 & 44 & 6 & 0.4048 \% \\ 9904 & 48 & 22 & 0.3533 \% \\ 2327 & 49 & 42 & 0.3511 \% \\ 2823 & 50 & 10 & 0.3454 \% \\ 2301 & 52 & 19 & 0.3331 \% \\ 2409 & 56 & 31 & 0.2787 \% \\ 2354 & 57 & 40 & 0.2779 \%\end{array}$

Table 3 reveals the sequence of constituent stocks, market capitalization ratios, and relationship coefficients. This article ranks 43 samples in order. Tsmc ranks first in stock weight, and its market capitalization accounts for $19.298 \%$ of TAIEX, but it ranks 21 st in the relationship coefficient sequence. Hon, which ranks second in the weighted ratio, ranks 15 th in the relationship coefficient sequence. For investors in many foreign institutions, or when the MSCI index is adjusted quarterly, the ranking of stock weights has always been one of the most important reference indicators, However, if the futures and spot transactions are paired according to this, there will be a phenomenon that performance is not synchronized or the effect of hedging is not obvious. Because the ranking of the relationship coefficient series is related to the synchronization with the index.

The ranking of stock weights generally represents the ranking of their importance in the market, but their stock price trends are not synchronized with the index. This result is confusing for many investors. In addition to this phenomenon in the top two stocks above, it also occurs in many heavyweight weight samples. For example, the stock champion Largan Precision ranks inversely in the relationship coefficient sequence. Formosa Plastics, the most important in the plastics industry, has a corresponding rank of 2 nd in the correlation coefficient sequence. Cathay and Fubon rank the top two in financial stocks, ranking 26th and 13th. Nan-Ya Plastics ranks sixth in the weighted ratio series but ranks first in the correlation coefficient series. Although this result is not strange or deviates from the rules of thumb used by investors, NanYa Plastics is not the focus of attention in the market, after all, therefore, such research results and findings are still helpful to clarify the problem of synchronization between the index and the stock price.

There are many investment tools in the financial market, and futures and stocks are the key financial tools that attract the most investors to participate. In order to obtain the best investment performance, investors are most interested in the topic of improving trading timing and predicting the future trend of stock prices. Through proper observation of some indicators to predict the stock price trend, and the ability to grasp the best timing of buying and selling stocks, under the blessing of the two can help investors obtain special returns. In order to find out the cycle of price rise and fall, if you can find appropriate observation indicators is one of the best methods. For example, after finding the stocks with the highest synchrony with the index, there are two strategies: First, if you observe the trend of individual stocks, if you find that there has been a turning point, you can switch to the futures market to buy and sell the index; the second is that there is a turning point in the market index, you can make a profit by making long or shorting individual stocks (or options). By observing the indicators of synchronization, investors can adopt the best investment strategy in the face of a turbulent stock market and avoid blind and irrational investment behavior.

After confirming Nan-Ya Plastics, which ranks first in the relationship coefficient series, this article will verify the evidence of its synchronization in the historical trajectory of the stock market to confirm whether it can be used as an important indicator to observe the future trend of TAIEX.

First, the stock price trend chart of TAIEX and NanYa Plastics is presented through a form of technical analysis (price line). It is a line connecting all closing prices in the monthly trend line from January 2003 to December 2018.

Second, the stock price trend chart of the index and individual stocks is marked, and the date of each turning point is recorded in detail. After statistics, it is found that TAIEX has 97 turning points, while Nan-Ya Plastics has 86 turning points. After deducting the meaningless turning points of short-term volatility, 66 important turning points have been confirmed in the 16-year trend. 
Finally, compare the dates of all turning points and indicate the number of peak-to-valley turning points of the index and individual stocks on the same day. In the 66 time points that have gone through long and short movements, the two overlapped 43 turning points, and the match rate reached 65.15\%; therefore, Nan-Ya Plastics can be regarded as a synchronization indicator of TAIEX.The advantage of the grey correlation study is finding consistency indicators. Therefore, by observing Nan-Ya Plastics (sample), this paper can infer the trend of the TAIEX (population).

\subsection{Other evidence of association research}

In order to avoid the bias of the data caused by sampling, in addition to the data during the above sample period, this study increased the sampling of the three sample periods under the factor that the number of samples could be increased and respectively calculated the sequence of the gray correlation coefficient. This shows that the gray correlation coefficient of South Asia Plastics ranks first in all the sampling intervals. This evidence shows that there is no sampling bias in the results of this study.

\section{CONCLUSION}

This paper provides five major contributions to our understanding of how the index is associated with the stocks. First, there is no positive correlation between the correlation coefficient sequence and the weight value of the constituent stocks. Secondly, the company with the highest synchronization with TAIEX is Nan-Ya Plastics; it can be regarded as a synchronization indicator of TAIEX. Third, through multiple sample period verifications, the association between Nan-Ya Plastics and TAIEX remains foremost. Fourth, this result is unchanged in the verification of the statistical method-correlation coefficient. Finally, the stock with the highest correlation with the index is ranked eighth, and the result doesn't substantially differ from the rule of thumb used by many investors. Although this result is not strange or deviates from the rules of thumb used by investors, Nan-Ya Plastics is not the focus of attention in the market, after all, therefore, such research results and findings are still helpful to clarify the problem of synchronization between the index and the stock price.

\section{References}

1. Lee, K.H.; \& Jo, G. S. (1999). Expert system for predicting stock market timing using A candlestick chart. Expert System with Applications, 16, 357-364.

2. Fayyad, U., Piatesky-Shapiro, G., Smyth, P., \& The, K. D. D. (1996). Process for extracting useful knowledge from volumes of data. ACM Comm., 39(11), 27-34.

3. Widom, J. (1995). Research problems in data warehousing. Proceedings of the Fourth International Conference on Information and
Knowledge Management, Baltimore, 25-30 November.

4. Demarest, M. (1994). Building the data mart. DBMA Magazine, 7 (8), 44-45.

5. Liu, H., \& Setiono, R. (1996). Dimensionality reduction via discretization.Knowledge-Based System, 9(1), 67-72..

6. Deng, J. (1982). Control problems of grey system. Systems \& Control Letters, 1(5),288-294.

7. Deng, J. (1989a). Introduction to grey system theory. The Journal of Grey Systems, 1(1),1-24.

8. Chan, K. and A. Hameed (2006). "Stock price synchronicity and analyst coverage in emerging markets." Journal of Financial Economics 80(1): $115-147$

9. Gul, F. A., et al. (2010). "Ownership concentration, foreign shareholding, audit quality, and stock price synchronicity: Evidence from China." Journal of Financial Economics 95(3): 425-442.

10. Hasan, I., et al. (2014). "Institutional development and stock price synchronicity: Evidence from China." Journal of Comparative Economics 42(1): 92-108.

11. Kim, Y., et al. (2020). "The Effect of Trade Secrets Law on Stock Price Synchronicity: Evidence from the Inevitable Disclosure Doctrine." The Accounting Review: 0000-0000.

12. Qiu, B., et al. (2019). "Trust and stock price synchronicity: evidence from China." Journal of Business Ethics: 1-13. 